\title{
On stability of odd-frequency superconducting state
}

\author{
Dmitry Solenov, Ivar Martin, Dmitry Mozyrsky \\ Theoretical Division (T-4), Los Alamos National Laboratory, Los Alamos, NM 87545, USA
}

(Dated: October 30, 2018)

\begin{abstract}
Odd-frequency pairing mechanism of superconductivity has been investigated for several decades. Nevertheless, its properties, including the thermodynamic stability, have remained unclear. In particular, it has been argued that the odd-frequency state is thermodynamically unstable, has an unphysical (anti-) Meissner effect, and thus can not exist as a homogeneous equilibrium phase. We argue that this conclusion is incorrect because it implicitly relies on the inappropriate assumption that the odd-frequency superconductor can be described by an effective Hamiltonian that breaks the particle conservation symmetry. We demonstrate that the odd-frequency state can be properly described within the functional integral approach using non-local-in-time effective action. Within the saddle point approximation, we find that this phase is thermodynamically stable, exhibits ordinary Meissner effect, and therefore can be realized as an equilibrium homogenous state of matter.
\end{abstract}

PACS numbers: 74.20.-z, 74.20.Mn, 74.20.Rp

Odd-frequency pairing mechanism was first introduced by Berezinskii ${ }^{1}$ in an attempt to describe the A-phase of superfluid ${ }^{3} \mathrm{He}$. Although, it was latter found that in the case of superfluid ${ }^{3} \mathrm{He}$ the pairing is odd is space ( $p$-wave) rather than in time, the question weather the same-spin fermions can develop anomalous correlations that are odd in frequency (" $p$-wave" along the time direction) remained open. The interest to this pairing mechanism was revived almost two decades later in the context of high-temperature superconductors by Balatsky and Abrahams, $\underset{2}{2}$ who generalized it to the case of opposite spin pairing. At the same time it has been argued that the odd-frequency superconducting order parameter does not correspond to a thermodynamically stable phase,,$\frac{3,4}{}$ and thus may not be realized as a homogenous equilibrium state of matter $\stackrel{5,6}{*}$ On the other hand, the authors of Ref. 7 have shown that path integral formulation of the problem can lead to the opposite conclusion. No resolution of this contradiction have been proposed so far. In this report we demonstrate that odd-frequency superconductor is thermodynamically stable and exhibits ordinary Meissner effect.

The argument on instability of the odd-frequency superconductor can be summarized as follows 3.8 . Thermodynamic stability is determined by the change of free energy in the ordered phase, relative to the disordered one. Near the second-order transition point it can be written $\mathrm{as}^{3,9}$

$$
\delta \Omega \sim-\frac{1}{\beta} \sum_{\omega, \mathbf{q}} \frac{\Delta(\omega, \mathbf{q}) \Delta^{+}(\omega, \mathbf{q})}{\omega^{2}+\xi_{\mathbf{q}}^{2}}
$$

with gap-functions $\Delta(\omega, \mathbf{q})$ and $\Delta^{+}(\omega, \mathbf{q})$ related to the anomalous (Matsubara) Green's functions $F(\omega, \mathbf{q})=\int d \tau e^{i \omega \tau}\left\langle\mathrm{T}_{\tau} c_{\mathbf{q}}(\tau) c_{-\mathbf{q}}(0)\right\rangle$ and $F^{+}(\omega, \mathbf{q})=$ $\int d \tau e^{i \omega \tau}\left\langle\mathrm{T}_{\tau} c_{-\mathbf{q}}^{\dagger}(\tau) c_{\mathbf{q}}^{\dagger}(0)\right\rangle$ through a self-consistency relation

$$
\Delta(\omega, \mathbf{q})=\sum_{\omega^{\prime}, \mathbf{q}^{\prime}} D\left(\omega-\omega^{\prime}, \mathbf{q}-\mathbf{q}^{\prime}\right) F\left(\omega^{\prime}, \mathbf{q}^{\prime}\right)
$$

and an identical relation for $F^{+}$and $\Delta^{+}$. Here $D(\omega, \mathbf{q})$ is irreducible interaction between quasiparticles with energies $\xi_{\mathbf{q}}$ (we assume that $D$ is real and even in both $\omega$ and $\mathbf{q}$ ), and $\beta$ is the inverse temperature. Here and in the following we consider spinless fermions - extension to the usual two-spin species case is straightforward and irrelevant for our purpose.

The sign of $\delta \Omega$ can be determined by analyzing the relation between $\Delta(\omega, \mathbf{q})$ and $\Delta^{+}(\omega, \mathbf{q})$. The non-zero averages $\left\langle\mathrm{T}_{\tau} c_{\mathbf{q}}(\tau) c_{-\mathbf{q}}(0)\right\rangle$ and $\left\langle\mathrm{T}_{\tau} c_{\mathbf{q}}^{\dagger}(\tau) c_{-\mathbf{q}}^{\dagger}(0)\right\rangle$ can only be obtained if they are taken with respect to a state with broken U(1)-symmetry (particle number conservation). If an appropriate broken-symmetry mean-field Hamiltonian $H_{M F}$ exists, then

$$
\begin{aligned}
F(\tau, \mathbf{q}) & =(1 / Z) \operatorname{Tr}\left[e^{-\beta H_{M F}} \mathrm{~T}_{\tau} e^{\tau H_{M F}} c_{\mathbf{q}} e^{-\tau H_{M F}} c_{-\mathbf{q}}\right],(3) \\
F^{+}(\tau, \mathbf{q}) & =(1 / Z) \operatorname{Tr}\left[e^{-\beta H_{M F}} \mathrm{~T}_{\tau} e^{\tau H_{M F}} c_{-\mathbf{q}}^{\dagger} e^{-\tau H_{M F}} c_{\mathbf{q}}^{\dagger}\right],
\end{aligned}
$$

where $Z=\operatorname{Tr}\left[e^{-\beta H_{M F}}\right]$. A straightforward comparison shows that the two Green's functions are related as

$$
F^{*}(\tau, \mathbf{q})=F^{+}(\tau, \mathbf{q}) \text {, or } F^{*}(\omega, \mathbf{q})=F^{+}(-\omega, \mathbf{q}) \text {. }
$$

As a consequence of Eq. (2) the functions $\Delta(\omega, \mathbf{q})$ and $\Delta^{+}(\omega, \mathbf{q})$ obey identical relation and therefore the product $\Delta(\omega, \mathbf{q}) \Delta^{+}(\omega, \mathbf{q})$ in Eq. (1) is negative definite for the odd-frequency $\Delta(\omega, \mathbf{q})$ producing $\delta \Omega>0$. As the result, one is forced to conclude that the odd-frequency superconducting phase is thermodynamically unstable. $\frac{3}{-}$ This conclusion can also be reached if one uses the Green's functions obtained in original work by Berezinskii. 1 As a related issue, one also finds an unphysical Meissner effect (i.e., with the negative Meissner kernel) $\frac{8}{6}$ Later it was suggested that the odd-frequency state might exist as an inhomogeneous phase, where the order parameter is modulated at the microscopic level, $, 4,5,6$ or might be a manifestation of some composite (even-frequency) order parameter. ${ }^{10}$

In what follows, we show that relation (4) holds only for the even-frequency anomalous Green's function. Oddfrequency superconducting state changes this relation, 
and Eq. (4) is modified to $F^{*}(\tau, \mathbf{q})=-F^{+}(\tau, \mathbf{q})$. The problem with the reasoning outlined above is that it assumed existence of a mean-field Hamiltonian, $H_{M F}$. However, to account for the retardation effects, which are essential in the case of the odd-frequency state, effective Hamiltonian language, e.g. Eq. (3), is inappropriate. In other words, $H_{M F}$ for odd-frequency superconductivity does not exist. Instead, one should consider an effective action that is essentially non-local in time.

To study the superconducting phase, we represent the partition function of the system is a functional integral11

$$
Z=\int \mathcal{D} \bar{\psi} \mathcal{D} \psi \mathcal{D} \Delta^{*} \mathcal{D} \Delta e^{-\mathcal{S}\left(\bar{\psi}, \psi, \Delta^{*}, \Delta\right)},
$$

with

$$
\begin{aligned}
S & =\int d x_{1} \bar{\psi}\left(x_{1}\right)\left[\partial_{\tau}+\hat{\xi}\right] \psi\left(x_{1}\right)+\int d x_{1} d x_{2} \frac{\left|\Delta\left(x_{1}, x_{2}\right)\right|^{2}}{D\left(x_{1}-x_{2}\right)} \\
& +\int d x_{1} d x_{2}\left[\Delta^{*}\left(x_{1}, x_{2}\right) \psi\left(x_{2}\right) \psi\left(x_{1}\right)+\Delta\left(x_{1}, x_{2}\right) \bar{\psi}\left(x_{1}\right) \bar{\psi}\left(x_{2}\right)\right]
\end{aligned}
$$

where $\bar{\psi}(x)$ and $\psi(x)$ are conjugate Grassmann variables corresponding to the fermionic fields $\psi^{\dagger}(\mathbf{r})=$ $\sum_{\mathbf{q}} e^{-i \mathbf{q} \mathbf{r}} c_{\mathbf{q}}^{\dagger}$ and $\psi(\mathbf{r})=\sum_{\mathbf{q}} e^{i \mathbf{q} \mathbf{r}} c_{\mathbf{q}}$, with $x$ labeling both spacial $\mathbf{r}$ and (Matsubara) time $\tau$ coordinates, and $\hat{\xi}$ is the kinetic energy operator, $\hat{\xi}=-\nabla_{\mathbf{r}}^{2} /(2 m)-\mu$. In Eqs. (5, 6) we have introduced the pairing field $\Delta\left(x_{1}, x_{2}\right)$ via the standard Hubbard-Stratonovich transformation 11 by decoupling the interaction term $\int d x_{1} d x_{2} D\left(x_{1}-\right.$ $\left.x_{2}\right) \bar{\psi}\left(x_{1}\right) \bar{\psi}\left(x_{2}\right) \psi\left(x_{2}\right) \psi\left(x_{1}\right)$. Note that up to this point, no approximation has been made.

As pointed out earlier, the anomalous Green's functions can be defined only with respect to a state with the broken $U(1)$ symmetry. Indeed it is easy to see that the quantity

$$
\int \mathcal{D} \bar{\psi} \mathcal{D} \psi \mathcal{D} \Delta^{*} \mathcal{D} \Delta \psi(x) \psi\left(x^{\prime}\right) e^{-\mathcal{S}\left(\bar{\psi}, \psi, \Delta^{*}, \Delta\right)}
$$

is identically zero: after integration over the $\Delta$ fields we average $\psi(x) \psi\left(x^{\prime}\right)$ with respect to the action containing only products $\bar{\psi} \psi$ of Grassmann variables. This average is nothing but $F\left(x-x^{\prime}\right)$ defined earlier in terms of the time-ordered average, which is indeed zero in normal phase. While in the normal phase, the partition function $Z$ of Eq. (5) is dominated by the vicinity of $\Delta=0$, below certain temperature the situation changes: The primary contribution to $Z$ comes from $\left|\Delta\left(x_{1}, x_{2}\right)\right| \neq 0$, which signals spontaneous $U(1)$ symmetry breaking. In addition, for spinless electrons, the superconducting state breaks either spatial parity (e.g. $p$-wave superconductor) or time reversal symmetry (odd-frequency superconductor). In the ordered state, one can expand the action in the vicinity of the non-zero saddle-point value of the order parameter $\Delta_{M F}\left(x_{1}-x_{2}\right)$, with mean field approximation corresponding to further neglecting the fluctuations around the saddle point. At the mean-field level, the anomalous correlation functions $\left(F\right.$ and $\left.F^{+}\right)$can be expressed as

$$
\begin{gathered}
F\left(\tau-\tau^{\prime} ; \mathbf{r}-\mathbf{r}^{\prime}\right)=Z_{M F}^{-1} \int \mathcal{D} \bar{\psi} \mathcal{D} \psi \psi(\mathbf{r}, \tau) \psi\left(\mathbf{r}^{\prime}, \tau^{\prime}\right) e^{-\mathcal{S}_{M F}} \\
F^{+}\left(\tau-\tau^{\prime} ; \mathbf{r}-\mathbf{r}^{\prime}\right)=Z_{M F}^{-1} \int \mathcal{D} \bar{\psi} \mathcal{D} \psi \bar{\psi}(\mathbf{r}, \tau) \bar{\psi}\left(\mathbf{r}^{\prime}, \tau^{\prime}\right) e^{-\mathcal{S}_{M F}}
\end{gathered}
$$

where

$$
\begin{aligned}
\mathcal{S}_{M F}= & \int d x_{1} \bar{\psi}\left(x_{1}\right)\left[\partial_{\tau}+\hat{\xi}\right] \psi\left(x_{1}\right) \\
& +\int d x_{1} d x_{2} \Delta_{M F}^{*}\left(x_{1}-x_{2}\right) \psi\left(x_{2}\right) \psi\left(x_{1}\right) \\
& +\int d x_{1} d x_{2} \Delta_{M F}\left(x_{1}-x_{2}\right) \bar{\psi}\left(x_{1}\right) \bar{\psi}\left(x_{2}\right),
\end{aligned}
$$

and $\Delta_{M F}\left(x_{1}-x_{2}\right)$ is again defined by the self-consistency condition, Eq. (2).

Now we are ready to determine the relation between $F$ and $F^{+}$. Due to the long history of the problem, and since we believe that this relation is the root of the divergent claims about the fate of the odd-frequency superconductivity, we present here all technical details. First, let us take the complex conjugate of $F$ :

$$
F^{*}\left(\tau-\tau^{\prime} ; \mathbf{r}-\mathbf{r}^{\prime}\right)=Z_{M F}^{-1} \int \mathcal{D} \bar{\psi} \mathcal{D} \psi \bar{\psi}\left(\mathbf{r}^{\prime}, \tau^{\prime}\right) \bar{\psi}(\mathbf{r}, \tau) e^{-\mathcal{S}_{M F}^{*}}
$$

where

$$
\begin{aligned}
& \mathcal{S}_{M F}^{*}=\int d x\left\{\partial_{\tau} \bar{\psi}(x) \psi(x)+\hat{\xi} \bar{\psi}(x) \psi(x)\right\} \\
& +\int d x_{1} d x_{2}\left[\Delta_{M F}\left(x_{1}-x_{2}\right) \bar{\psi}\left(x_{1}\right) \bar{\psi}\left(x_{2}\right)\right. \\
& \left.+\Delta_{M F}^{*}\left(x_{1}-x_{2}\right) \psi\left(x_{2}\right) \psi\left(x_{1}\right)\right] .
\end{aligned}
$$

Integrating the first two terms in Eq. (12) by parts we obtain $\int d x \bar{\psi}(x)\left[-\partial_{\tau}+\hat{\xi}\right] \psi(x)$. Then defining the new variables according to $\bar{\eta}(\mathbf{r}, \tau)=\bar{\psi}(\mathbf{r},-\tau)$ and $\eta(\mathbf{r}, \tau)=$ $\psi(\mathbf{r},-\tau)$, and changing $\tau \rightarrow-\tau$ in every integral in Eq. (12), we obtain

$$
F^{*}\left(\tau-\tau^{\prime} ; \mathbf{r}-\mathbf{r}^{\prime}\right)=Z_{M F}^{-1} \int \mathcal{D} \bar{\eta} \mathcal{D} \eta \bar{\eta}\left(\mathbf{r}^{\prime},-\tau^{\prime}\right) \bar{\eta}(\mathbf{r},-\tau) e^{-\tilde{\mathcal{S}}_{M F}}
$$

with

$$
\begin{aligned}
& \tilde{\mathcal{S}}_{M F}=\int d x \bar{\eta}(x)\left[\partial_{\tau}+\hat{\xi}\right] \eta(x) \\
& +\int d x_{1} d x_{2}\left[\Delta_{M F}^{*}\left(\tau_{2}-\tau_{1}, \mathbf{r}_{1}-\mathbf{r}_{2}\right) \eta\left(x_{2}\right) \eta\left(x_{1}\right)\right. \\
& \left.+\Delta_{M F}\left(\tau_{2}-\tau_{1}, \mathbf{r}_{1}-\mathbf{r}_{2}\right) \bar{\eta}\left(x_{1}\right) \bar{\eta}\left(x_{2}\right)\right]
\end{aligned}
$$

For even-in- $\tau \Delta(\tau, \mathbf{r})$, (e.g., $p$-wave for a single spin species case) we have $\tilde{\mathcal{S}}_{M F}=\mathcal{S}_{M F}$ and therefore comparing Eqs. (13, 14) with Eqs. (9, 10) we recover Eq. (4). 
For odd-in- $\tau \Delta(\tau, \mathbf{r})$, (e.g., $s$-wave with a single spin species) we see that by changing $\tau \rightarrow-\tau$ a minus sign is generated in the last two terms in the RHS of Eq. (14) as compared to Eq. (12). This difference can be removed by another change of variables $\eta(\mathbf{r}, \tau) \rightarrow i \eta(\mathbf{r}, \tau)$, $\bar{\eta}(\mathbf{r}, \tau) \rightarrow-i \bar{\eta}(\mathbf{r}, \tau)$, which is a simple gauge transformation. We obtain again $\tilde{\mathcal{S}}_{M F}=\mathcal{S}_{M F}$. However, an additional factor (-1) now appears before the entire path integrals owning to the fact that the quantity $\bar{\eta} \bar{\eta}$ transforms into $(-\bar{\eta} \bar{\eta})$ as a result of the last gauge transformation. Therefore comparing Eqs. (13, 14) with Eqs. (9) 10) we finally obtain that in the odd-frequency case we have the relation

$$
F^{*}\left(\tau-\tau^{\prime}, \mathbf{r}-\mathbf{r}^{\prime}\right)=-F^{+}\left(\tau-\tau^{\prime}, \mathbf{r}^{\prime}-\mathbf{r}\right)
$$

or

$$
F^{*}(\omega, \mathbf{q})=F^{+}(\omega, \mathbf{q})
$$

Obviously Eq. (16) holds for the even-frequency case as well - in which case $F^{+}(-\omega, \mathbf{q})$ in Eq. (44) can be replaced by $F^{+}(\omega, \mathbf{q})$ for even-frequency $F$. As a result, contrary to the conclusion of Refs. 3, $4,5,6$, the product $\Delta(\omega, \mathbf{q}) \Delta^{+}(\omega, \mathbf{q})$ in Eq. (11) is positive definite both for odd- and even-frequency pairing and therefore $\delta \Omega<0$. The same conclusion can be reached by directly analyzing the mean-field free energy, given by Eqs. (5) 6), as the system undergoes the phase transition. Thus we conclude that the odd-frequency superconducting phase has free energy lower than that of the normal phase. The magnitude of the order parameter has to be determined from the self-consistency condition and is non-zero below the superconducting transition temperature.

It can be also verified that due to relation (16) oddfrequency superconducting phase has a positive Meissner kernel and therefore a physically meaningful Meissner effect. The supercurrent and the vector potential are related as $\mathbf{j}(\mathbf{k})=-\mathcal{K}(\mathbf{k}) \mathbf{A}(\mathbf{k})$, where the (Meissner) kernel $\mathcal{K}$ is expressed ${ }^{9}$ as

$$
\begin{aligned}
\mathcal{K}(\mathbf{k})=\frac{N e^{2}}{m} & +\frac{2 e^{2}}{m^{2} \beta} \sum_{\omega} \int \frac{d \mathbf{p}}{(2 \pi)^{3}} \mathbf{p}^{2}\left[\mathcal{G}\left(\omega, \mathbf{p}_{+}\right) \mathcal{G}\left(\omega, \mathbf{p}_{-}\right)\right. \\
& \left.+F\left(\omega, \mathbf{p}_{+}\right) F^{+}\left(\omega, \mathbf{p}_{-}\right)\right]
\end{aligned}
$$

Here $\mathbf{p}_{ \pm}=\mathbf{p} \pm \mathbf{k} / 2$. The Green's functions can be easily obtained from Eqs. (8, 9, 10). We have

$$
F(\omega, \mathbf{q})=\frac{2 \Delta_{M F}(\omega, \mathbf{q})}{\omega^{2}+\xi(\mathbf{q})^{2}+4\left|\Delta_{M F}(\omega, \mathbf{q})\right|^{2}}
$$

$$
\begin{gathered}
F^{+}(\omega, \mathbf{q})=\frac{2 \Delta_{M F}^{*}(\omega, \mathbf{q})}{\omega^{2}+\xi(\mathbf{q})^{2}+4\left|\Delta_{M F}(\omega, \mathbf{q})\right|^{2}} \\
\mathcal{G}(\omega, \mathbf{q})=\frac{i \omega+\xi(\mathbf{q})}{\omega^{2}+\xi(\mathbf{q})^{2}+4\left|\Delta_{M F}(\omega, \mathbf{q})\right|^{2}}
\end{gathered}
$$

Note that the form of Eqs. (18, 19) is consistent with Eq. (16), not with Eq. (4). Had we used Eq. (4), we would have obtained $\Delta_{M F}^{*}(-\omega, \mathbf{q})$ in Eq. (19) as well as $-|\Delta(\omega, \mathbf{q})|^{2}$ in the denominators of Eqs. (18, 19, 20).

As usual Eq. (17) is divergent and we regularize it by subtracting $\mathcal{K}(\mathbf{k})$ for $\Delta_{M F}(\omega, \mathbf{q})=0$ (obviously $\mathcal{K}(\mathbf{k})=0$ in normal phase) $\stackrel{9}{\underline{9}}$ In the long wave-length limit and for $\Delta_{M F}(\omega, \mathbf{q})$ independent of $\mathbf{q}$ (i.e., for pairing in $s$-wave channel) we obtain

$$
\mathcal{K}(\mathbf{k} \rightarrow 0)=\frac{\pi N e^{2}}{m \beta} \sum_{\omega} \frac{4\left|\Delta_{M F}(\omega)\right|^{2}}{\left(\omega^{2}+4\left|\Delta_{M F}(\omega)\right|^{2}\right)^{3 / 2}} .
$$

This equation obviously is positive definite. Note that if we had used Eq. (4) (which is invalid as we argue above), we would have obtained $\Delta^{+}(\omega, \mathbf{q}) \Delta(\omega, \mathbf{q})=$ $\Delta^{*}(-\omega, \mathbf{q}) \Delta(\omega, \mathbf{q})$ in the numerator in the RHS of Eq. (21) and thus negative Meissner kernel for the oddfrequency case.

While possessing similar electromagnetic properties (i.e., the Meissner effect) to its familiar even-frequency counterpart, the odd-frequency superconductor is expected to differ from the even-frequency one in several important aspects. Here we mention just some of them qualitatively. The equal spin pairing considered above, leads to a gapless superconductor, with an isotropic $(s-$ wave) electronic spectral function. The only other known example where this can happen is the $s$-wave superconductor with a relatively high concentration of magnetic impurities; however, for odd-frequency superconductor this would occurs even in the clean case. The oddfrequency anisotropic $s$-wave superconductor can readily exceed the Pauli paramagnetic limit, and can show very little change in magnetic susceptibility (Knight shift) across the superconducting transition. These and other properties, in particular the manifestations of the timereversal symmetry braking in such superconductors, are attractive direction for future detailed theoretical investigations.

We acknowledge valuable and stimulating discussions with E. Abrahams and A. Balatsky. This work is supported by the US DOE.
1 V. L. Berezinskii, Pis'ma Zh. Eksp. Teor. Fiz 20, 628 (1974) [JETP Lett. 20, 287 (1974)].

2 A. Balatsky and E. Abrahams, Phys. Rev. B 45, 13125 (1992).

3 R. Heid, Z. Phys. B 99, 15 (1995).
4 D. L. Cox, A. Zawadowski, Adv. Phys. 47, 599 (1998).

${ }^{5}$ P. Coleman, E. Miranda, and A. Tsvelik, Phys. Rev. B 49, 8955 (1994).

${ }^{6}$ R. Heid, Ya. B. Bazaliy, V. Martisovits, D. L. Cox, Phys. Rev. Lett. 74, 2571 (1995). 
7 D. Belitz, T. R. Kirkpatrick, Phys. Rev. B 58, 8214 (1998); D.Belitz, T. R. Kirkpatrick, Phys. Rev. B 60, 3485 (1999).

8 E. Abrahams, A. V. Balatsky, private comminication.

9 A. A. Abrikosov, L. P. Gorkov, and I. E. Dzyaloshinski, Methods of quantum field theory in statistical physics (Dover, 1975).
10 E. Abrahams, A. Balatsky, D. J. Scalapino, J. R. Schrieffer, Phys. Rev. B 52, 1271 (1992).

11 J. W. Negele, H. Orland, Quantum many-particle systems (Addison-Wesley Publishing Company, 1988). 